\title{
CFPU: A Cell-Free Processing Unit for High-Throughput, Automated In Vitro Circuit Characterization in Steady-State Conditions
}

\author{
Zoe Swank (D) and Sebastian J. Maerkl $(\mathbb{D}$ \\ Institute of Bioengineering, School of Engineering, École Polytechnique Fédérale de Lausanne, Switzerland \\ Correspondence should be addressed to Sebastian J. Maerkl; sebastian.maerkl@epfl.ch
}

Received 13 December 2020; Accepted 24 February 2021; Published 17 March 2021

Copyright (c) 2021 Zoe Swank and Sebastian J. Maerkl. Exclusive Licensee Nanjing Agricultural University. Distributed under a Creative Commons Attribution License (CC BY 4.0).

\begin{abstract}
Forward engineering synthetic circuits are at the core of synthetic biology. Automated solutions will be required to facilitate circuit design and implementation. Circuit design is increasingly being automated with design software, but innovations in experimental automation are lagging behind. Microfluidic technologies made it possible to perform in vitro transcription-translation (tx-tl) reactions with increasing throughput and sophistication, enabling screening and characterization of individual circuit elements and complete circuit designs. Here, we developed an automated microfluidic cell-free processing unit (CFPU) that extends highthroughput screening capabilities to a steady-state reaction environment, which is essential for the implementation and analysis of more complex and dynamic circuits. The CFPU contains 280 chemostats that can be individually programmed with DNA circuits. Each chemostat is periodically supplied with tx-tl reagents, giving rise to sustained, long-term steady-state conditions. Using microfluidic pulse width modulation (PWM), the device is able to generate tx-tl reagent compositions in real time. The device has higher throughput, lower reagent consumption, and overall higher functionality than current chemostat devices. We applied this technology to map transcription factor-based repression under equilibrium conditions and implemented dynamic gene circuits switchable by small molecules. We expect the CFPU to help bridge the gap between circuit design and experimental automation for in vitro development of synthetic gene circuits.
\end{abstract}

\section{Introduction}

Engineering gene regulatory networks from the bottom-up is a cornerstone of synthetic biology, providing a means to decipher natural biological systems [1-3] and develop new applications [4-6]. Developing new circuit designs in vivo requires design-build-test cycle iterations, where every iteration requires time-consuming molecular cloning steps. In vitro tx-tl systems on the other hand have proved to be powerful tools for accelerating the design-build-test cycle [3, 7-10]. Recent technological advancements increased complexity and throughput of in vitro tx-tl experiments, such as the use of acoustic liquid handling robots [11, 12], or various microfluidic platforms [13-15]. Although these methods facilitated detailed studies of molecular circuits, they remain limited because of their use of simple batch reactions.

Steady-state tx-tl reactions have been realized on microfluidic devices to enable in vitro implementation of dynamic circuits $[16,17]$. These methods vastly expanded the type and complexity of networks that can be run in an in vitro environment compared to what is possible using batch reactions. However, these first-generation chemostat devices are limited in throughput, creating a need for improved platforms that can carry out steady-state tx-tl reactions at higher capacities. We developed a microfluidic device that combines the high-throughput capacities of batch reaction devices [15] and the sophistication of microfluidic chemostats $[16,17]$ to perform steady-state tx-tl reactions in high throughput.

In addition to being able to perform high-throughput, steady-state tx-tl reactions, we developed a method to formulate reagent compositions on the fly to automatically screen condition space or to dynamically perturb the system for analysis. Fluidic automation and process integration are increasingly recognized as being important capabilities for both chemical $[18,19]$ and biological $[20,21]$ methods. To enable fluidic process automation and dynamic, on-the-fly 
process changes, we integrated a computer-controlled fluidic metering strategy using microfluidic pulse width modulation (PWM) [22].

The resulting microfluidic CFPU enables the automated exploration of parameter space in 280 parallel running steady-state reactions. Spotting and immobilizing DNA templates to program each chemostat allow screening of a large DNA sequence or concentration space. Furthermore, with the integration of computer-controlled microfluidic PWM for on-chip metering and mixing of reaction components, the platform can perform fully automated and complex fluid processing tasks $[22,23]$. By using different chemostat geometries, based on the design of Karzbrun et al. [17], we can alter reagent diffusion times. We integrated programmable DNA arrays, controlled DNA surface immobilization, highly parallel chemostats, and programmable microfluidic PWM into a single CFPU and demonstrate its functionality by mapping the concentration and sequence space of transcription factor-based repression at a steady state and implemented a genetic toggle switch network.

\section{Results}

2.1. Design and Characterization of the CFPU. The two-layer PDMS microfluidic device is fabricated using multilayer softlithography [24] and consists of a control and a flow layer, whereby fluids on the flow layer can be manipulated by applying pressure to the control lines (Figure 1(a)). The flow layer contains 280 unit cells, or chemostats, that can each run a unique and steady-state $\mathrm{tx}$ - $\mathrm{tl}$ reaction (Figure $1(\mathrm{~b})$ ). Each chemostat is connected to an exchange channel through which fresh reagents are flowed. The length of the connecting channel ranges from $50 \mu \mathrm{m}$ to $450 \mu \mathrm{m}$ and a fixed width of $25 \mu \mathrm{m}$. The flow layer inlets were optimized for mixing reagents by PWM [22]. PWM is employed in electrical systems to regulate voltage levels. Similarly, microfluidic PWM involves the generation of alternating pulses of different solution inputs that then mix together via diffusion to generate defined concentration levels depending on the relative duration each solution is flowed. The inlet flow channels are all of equal length as are the lengths of the control lines that regulate the top five inlets, ensuring the PWM module accurately meters reagents. We incorporated a serpentine channel after the PWM module to allow reagent plugs formed by PWM to mix before reaching the beginning of the exchange channels.

The device is prepared by first spotting fluorescently labeled and biotinylated linear DNA templates onto an epoxy-coated glass slide using a microarray robot (Figure 1(c)). The PDMS chip is then aligned on top of the DNA microarray and bonded to the glass. After bonding, the glass surface of the microfluidic unit cells, with the exception of the spotting chamber, which is the circular region below valve \#5 (Figure 1(b)), is patterned with biotin-BSA and neutrAvidin, resulting in a circular region of neutrAvidin coating (Figure 1(d)), to which biotinylated DNA can bind. Throughout this process, the DNA spot remains isolated in the lower chamber, sealed off by the neck valve. Once surface patterning is completed, the DNA spot is solubilized, allowing the DNA to diffuse into the upper part of the unit cell and bind to the neutrAvidin-coated area (Figure 1(e)). Any unbound DNA is then washed from the device, and tx-tl extract is introduced into the exchange channel by mixing the energy and lysate solutions directly on-chip with the PWM module (Figure 1(f)). While the exchange channel is being replaced with fresh extract, the bottom half of the unit cell is isolated with a valve that is then released to allow diffusion of fresh reagents into the unit cell. Another set of valves is actuated to isolate the unit cells from each other during incubation. The process of flowing fresh cell-free extract followed by an incubation step is repeated for up to 20 hours and achieves continuous, long-term gene expression (Figure 1(g)). By periodically flowing fresh extract, we are able to use far smaller reagent volumes $(\sim 3 \mu \mathrm{L} / \mathrm{h})$ or approximately 20x less than previous methods $(\sim 60 \mu \mathrm{L} / \mathrm{h})$ [17]. Additionally, on-chip mixing of lysate and energy components eliminates the need to cool the premixed reagents offchip [25]. We postulate that separating the protein components in the cell lysate from the energy solution prevents off-target reactions that deplete the concentration of small energy molecules. Using the multiplexing (MUX) valves (Figure 1(a)), each row of the CFPU can be addressed with a specific tx-tl reagent composition (Figure 1(h)). For instance, up to eight different concentrations of a transcription factor (TF) can be combined with the tx-tl components and tested in combination with different DNA template concentrations or DNA promoter variants. The added reagent, such as purified proteins or small molecules, is automatically formulated on-chip according to user-defined time and concentration parameters, enabling dynamic gene circuit studies (Figure 1(i)).

To confirm that the tx-tl extract solutions could be adequately mixed on-chip, we tested various PWM cycle periods with duty cycles fixed at $50 \%$. We used mCherry and GFP as tracers $(\sim 27 \mathrm{kDa})$ in the energy and lysate solutions, respectively, and monitored on-chip mixing at different locations downstream of the inlets. We chose to use fluorescent proteins as tracers to serve as a proxy for the protein components in the lysate, which are important for tx-tl. Although certain biomolecules, including the ribosomes, are larger than GFP, we can at least be sure that the mixing of the two solutions is homogeneous for most of the protein components. Directly after the inlets, the energy solution and lysate plugs can be clearly visualized for cycle periods ranging from 600 to $1400 \mathrm{~ms}$ (Figure S1A). After passing through the serpentine channel, the two solutions have mixed for cycle periods less than $800 \mathrm{~ms}$, and at the beginning of the exchange channel, mixing is complete for cycle periods up to $1200 \mathrm{~ms}$ long (Figure S1B, C). As the CFPU architecture is separated into eight different rows, we also checked whether mixing is uniform across all rows and confirmed that for cycle periods of 600 and $1000 \mathrm{~ms}$, uniform mixing is achieved across all rows of the device (Figure S1D, E).

Given that $\mathrm{tx}$-tl solutions could be effectively mixed onchip, we next tested steady-state gene expression to characterize the effect of the different unit cell geometries (Figure 2(a)). For a given DNA template concentration, we observed a range of steady-state expression levels depending on connecting channel length (Figure 2(b)). This steady state 


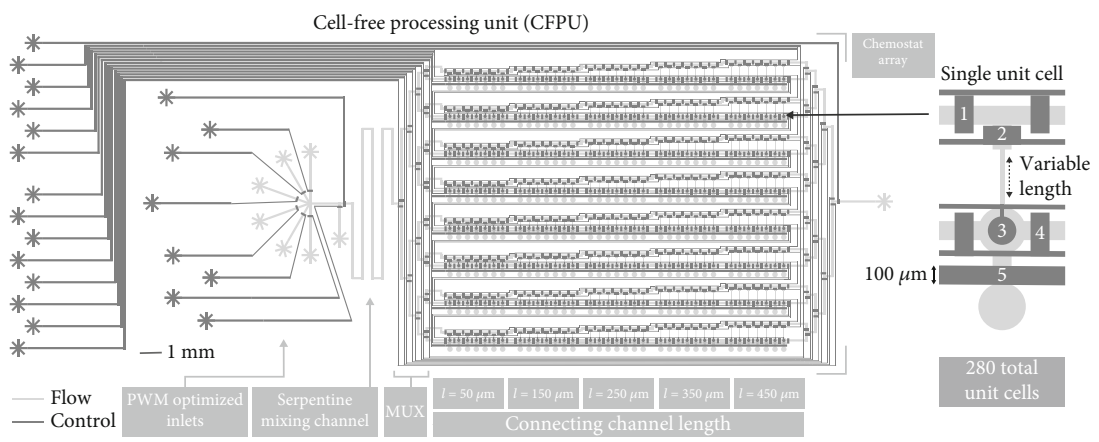

(a)

(b)

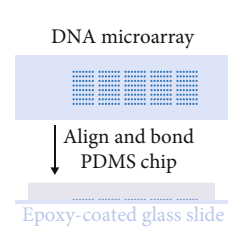

(c)

Experimental overview

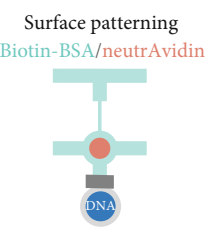

(d)

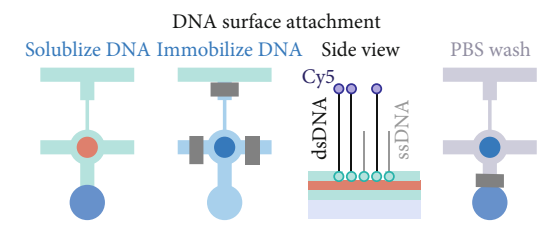

(e)

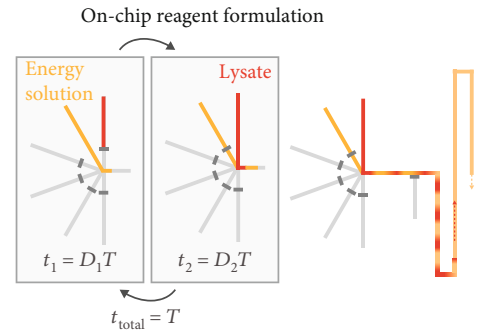

(f)

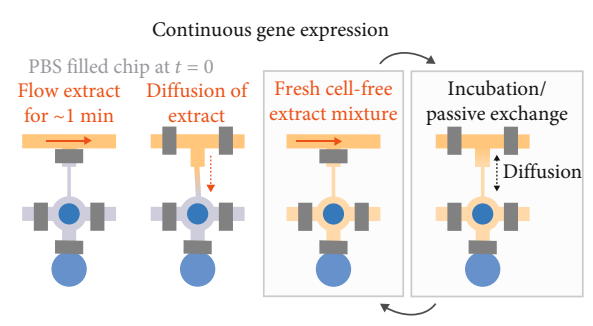

Total cycle time $=15$ minutes

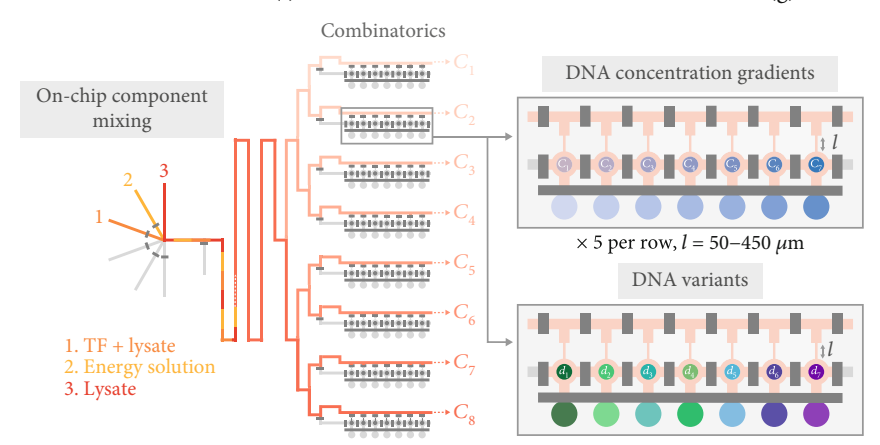

(h)

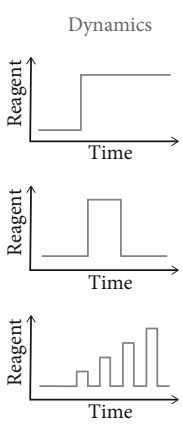

(i)

FIgURE 1: Overview of the CFPU architecture and functionality. (a) Illustration of the microfluidic CFPU design, highlighting the PWM optimized inlets, followed by the serpentine mixing channel and chemostat array with variable connecting channel lengths. (b) Design of a single unit cell. The pneumatic valves are described from top to bottom: (1) a pair of valves separate each unit cell in the exchange channel, (2) a valve prevents flow into the unit cell while the exchange channel is being replaced with fresh reagents, (3) the button valve enables surface patterning for DNA attachment, (4) sandwich valves separate the reaction chambers of each unit cell from one another, and (5) the neck valve isolates the DNA spot until surface patterning is complete. (c-g) A schematic summary of the experimental protocol: (c) DNA templates are spotted with a microarray robot onto an epoxy-coated glass slide, on top of which the PDMS chip is aligned. (d) The reaction chamber of the unit cell is patterned with biotin-BSA and neutrAvidin. (e) The DNA spot is solubilized, permitting the DNA to diffuse into the upper half of the unit cell and bind to the surface, after which all unbound DNA is washed away. (f) Lysate and energy solutions are then flowed and mixed on-chip with PWM. Each solution is flowed for a time $t=D T$, where $D$ and $T$ represent the duty cycle and cycle period, respectively. (g) Initially, a cell-free extract solution is flowed onto the chip and diffuses into a unit cell that is filled with PBS. Every 15 minutes, fresh cell-free extract is flowed through the exchange channel for $\sim 1$ minute followed by an incubation period that enables the exchange of reagents via diffusion. (h) Examples of combinatoric experiments that can be performed with this chip, including the generation of different concentrations of an input reagent using PWM. Each row of the device can be addressed with a specified reagent concentration, which is then combined with a range of DNA template concentrations or template variants, and different connecting channel lengths. (i) Reagent formulation is dynamic, and a given reagent can be introduced into the device at user-defined times, allowing dynamic perturbations to be performed. 


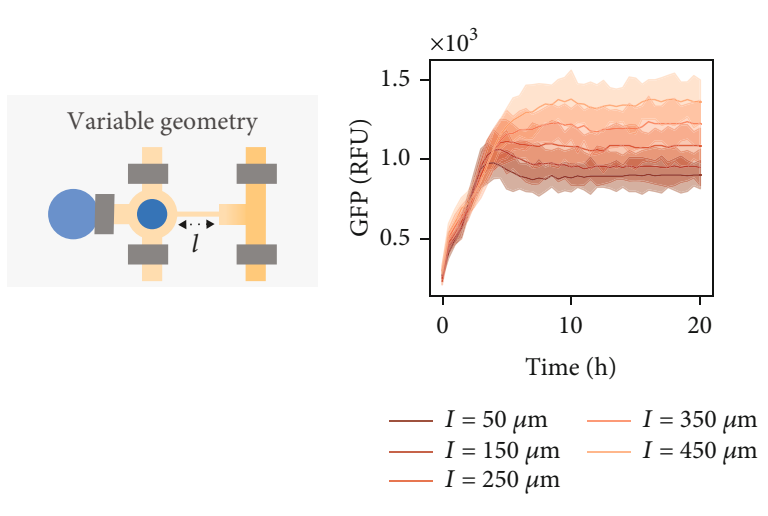

(a)

(b)

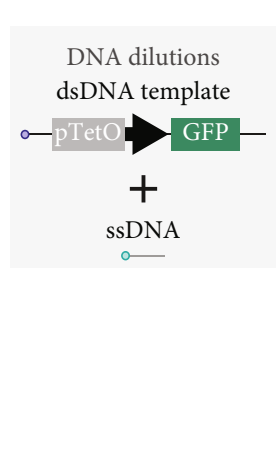

(c)

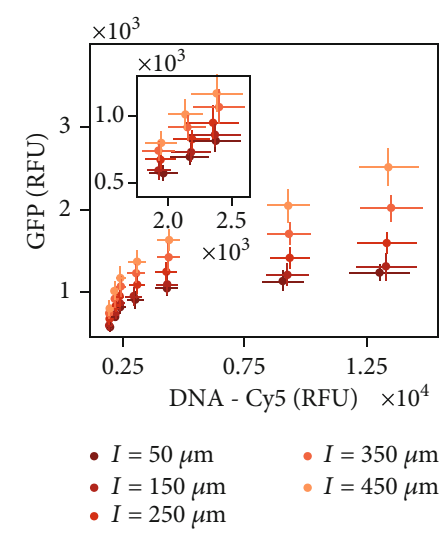

(d)

FIgURE 2: CFPU characterization and applications. (a) Protein expression using a single DNA template was carried out on-chip in order to characterize the effect of variable connecting channel lengths. (b) GFP expression versus time shown for a single DNA concentration and different connecting channel lengths. (c) DNA dilutions are made by mixing different ratios of dsDNA templates with short biotinylated ssDNA oligos. (d) The steady-state GFP expression level versus the Cy5 fluorescence signal of dsDNA template attached to the surface for all connecting channel lengths and DNA dilutions. The inlaid plot shows the three lowest DNA concentrations. The lines or points shown in $(b, d)$ represent mean values $\pm \mathrm{SD}(n=8)$.

can be disrupted by flowing PBS in place of the tx-tl extract mixture (Figure S2). An increase in connecting channel length correlates with an increase in steady-state GFP expression level as described previously [17]. Furthermore, the time required to reach steady-state levels increased for longer connecting channels. As described previously [15], binding of DNA templates on the surface of each unit cell and therefore their concentration can be precisely controlled by using a mixture of double-stranded DNA templates and single-stranded biotinylated DNA oligos (Figure 2(c)). When we varied the DNA template concentrations, we observed a wide range of GFP expression levels as expected (Figure 2(d)). Additionally, we achieved excellent chip-tochip reproducibility (Figure S3). Plotting GFP expression over time, we saw that the higher the DNA template concentration or the longer the connecting channel, the longer it took until a steady state was reached (Figure S4). Furthermore, at increased DNA concentrations, we observed a peak of GFP expression at the beginning, which later levels out. We hypothesize that for higher concentrations of DNA, the exchange rate of fresh reagents into the unit cell is not fast enough to maintain higher intensity steady-state expression. Similar dynamics were noted by Karzbrun et al. for nonregulated GFP expression, and they normalized for this effect. Nonetheless, at lower DNA template concentrations, steady states can be reached after $\sim 4-6$ hours for all connecting channel lengths. For this reason, we performed all following experiments with a dsDNA to ssDNA ratio below $1: 5$.

2.2. Mapping Steady-State Repression by Titrating Repressor Concentration. Forward engineering of gene regulatory networks often requires in-depth characterization of the transcription factors used to regulate the network. For instance, repression using synthetic zinc finger (ZF) transcriptional regulators [26] can be tuned by varying both the concentration of $\mathrm{ZF}$ in the tx-tl reaction, as well as by tuning the ZF binding site [15]. As one proof-of-concept application, we screened the effect of different ZF-TF concentrations on repression at a steady state.

We first tested whether it was possible to mix three different solutions with PWM: (1) a lysate solution containing a GFP tracer, (2) a plain lysate solution, and (3) an energy solution containing an mCherry tracer. As the ratio between the lysate and energy solution needs to be maintained at $1: 1$, the duty cycle of the energy solution is kept constant at $50 \%$, while the remaining $50 \%$ of the duty cycle is divided between the two lysate solutions to achieve varying concentrations of GFP. The minimum duty cycle used was $5 \%$, allowing enough time for the valves to fully open and close in the allotted time. For a cycle period of $1 \mathrm{~s}$, we observed a linear correlation between GFP concentration and duty cycle percentage (Figure S5). At the same time, the level of mCherry, which serves as a tracer in the energy solution, remained constant, indicating that the 1:1 ratio of energy and lysate solution was maintained. Up to ten dilutions could be generated for one stock solution of GFP. If a biomolecule of interest needs to be further diluted, as many as three stock solutions could be used, given the available number of PWM optimized inlets, leading to a significant increase in the dynamic range achievable.

After confirming that we could accurately mix three solutions on-chip with PWM, we designed an experiment to measure the steady-state repression for $Z F_{A A A}$ at various concentrations in combination with a linear DNA template comprised of a GFP gene downstream of a $\lambda$ PR promoter, containing two ZF binding sites (Figure 3(a)). We used a purified ZF-TF tagged with mScarlet, for on-chip visualization and quantification of protein concentration. PWM generated four ZF concentrations including a negative control and fed these into four clusters of rows using the MUX upstream of the exchange channels. Initially, the tx-tl reaction was brought to a steady state without ZF, and after 6.5 hours, the different ZF concentrations were added to the 


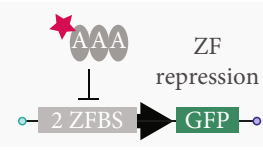

(a)

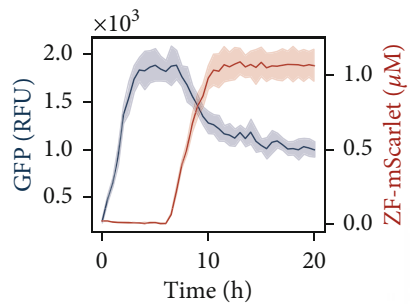

(b)

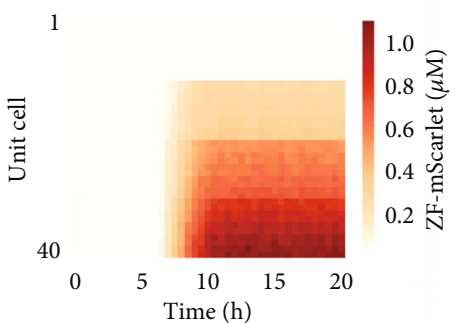

(c)

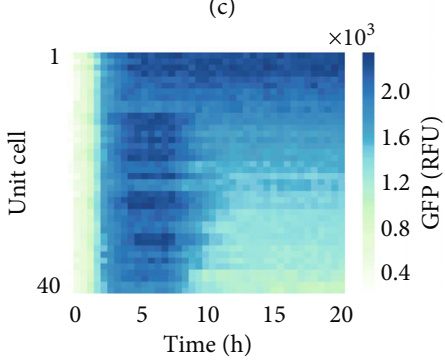

(d)

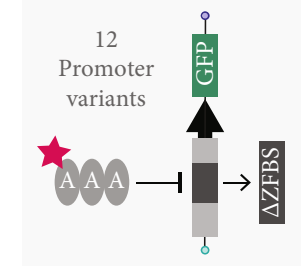

(e)



(f)

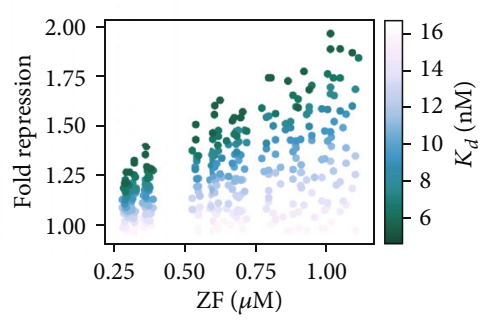

(g)

FIGURE 3: ZF combinatorics. (a) PWM is used to mix three components on-chip to generate different concentrations of an mScarlet-tagged ZF which is then screened against a target template with two ZF binding sites in the promoter. (b) Example of time-lapse traces for GFP expression and ZF-mScarlet signal. The lines represent the mean values $\pm \mathrm{SD}(n=2)$, where the SD is designated by the shaded regions. (c) ZF-mScarlet concentration over time for all unit cells containing a target template with two ZF binding sites in the promoter. (d) GFP expression versus time for the same unit cells shown in the preceding plot. (e) Multiple targets can be screened simultaneously on-chip, including promoter variants that contain mutated ZF binding sites. (f) Fold repression over time for targets containing modified ZF binding sites, shown in order of increasing $K_{d}$, with the wild type binding site sequence at the top. The fold repression is calculated by dividing the signal obtained from no added ZF by the signal obtained from the highest quantity of added ZF (duty cycle $=50 \%)$. The time-lapse data shown in this plot corresponds to unit cells with a connecting channel length of $l=50 \mu \mathrm{m}$. (g) All end point fold repression values calculated for each duty cycle and connecting channel length, plotted according to the ZF binding site $K_{d}$. Data shown in panels (f, g) was collected using two chips and represent the mean values $\pm \operatorname{SD}(n=2)$.

respective sets of rows on the chip. As an example, we show the GFP signal and ZF-mScarlet concentration over time in two unit cells with the same connecting channel length and addressed with the same ZF input concentration (Figure 3(b)). Looking at the ZF-mScarlet signal in each unit cell over time, we observe four distinct sections corresponding to the four ZF concentrations (Figure 3(c)). However, within each quadrant, we also notice a slight gradient, which is associated with the different connecting channel lengths. As the $\mathrm{ZF}$ diffuses into the unit cells, we begin to see a decrease in GFP expression (Figure 3(d)). We tested several promoter variants, containing one ZF binding site with different point mutations known to modulate repression to varying degrees (Figure 3(e)). Overall, four ZF concentrations were generated to test repression of 12 different DNA templates in five different unit cell geometries, for a total of 240 unique combinations, measured in replicates on two devices. The binding site mutations were chosen based on previously measured $K_{d} s$ [15], including two mutations that completely ablated repression $(\Delta 9 \mathrm{~A}, 5 \mathrm{~T})$ and nine mutations that covered the entire dynamic range between consensus and nonspecific binding. If we consider a single unit cell dimension, we see that the fold repression for different target templates decreases over time as $K_{d}$ associated with a given binding site increased (Figure $3(\mathrm{f})$ ). We observe a range of steady-state fold repression values depending on the 
concentration of $\mathrm{ZF}$ within a given unit cell and a correlation between all fold repression values, ZF concentration, and the measured binding affinity for a given promoter (Figure $3(\mathrm{~g})$ ). These results are in agreement with our previous batch analysis [15], and they provide a proof of concept that the microfluidic platform can be used to simultaneously screen target sequence space in conjunction with transcription factor concentrations in a steady-state tx-tl reaction.

\subsection{Implementing Dynamic Gene Circuits Controlled by} Small Molecules. After formulating different TF concentrations on our chip at predesignated times, we went on to test whether it was possible to implement and control dynamic gene circuits. We set up a simple repression assay whereby TetR can repress a GFP encoding template that includes the Tet operator in its promoter region, unless a small molecule, anhydrotetracycline (aTc), is present and prevents TetR from binding to the operator (Figure 4(a)). While holding the GFP DNA template concentration constant, we varied the concentration of TetR template and added a loading control template to maintain the same total DNA concentration. After allowing the reaction to reach steady state, we introduced aTc in a three-hour pulse. As the concentration of the TetR template was increased, we observed a corresponding decrease in the steady-state expression levels of GFP (Figure 4(b)). During the aTc pulse, repression was inhibited and GFP expression rose to the control level where no TetR template was present. Once aTc was no longer supplied to the unit cells, the GFP template was again repressed and the initial steady state reestablished. Furthermore, we observed that the reaction time to the aTc pulse differs according to the connecting channel length (Figure 4(c)).

As a final example, we combined the ability to introduce small molecule inputs with dynamic gene circuits in order to implement and control a genetic toggle switch [27]. The toggle switch is composed of two linear DNA templates that encode two repressor proteins, TetR and LacI, where each protein-encoding gene is downstream of mutually repressible promoters (Figure 4(d)). A transient pulse of isopropyl- $\beta$-Dthiogalactopyranoside (IPTG) or aTc causes the circuit to switch from one state to another. Using different concentrations of a Lac-repressible GFP reporter, we allowed the reaction to reach a steady state, observing increasing levels of the leak for higher concentrations of reporter template spotted. IPTG was introduced into the chip after 7 hours, causing TetR and GFP to be derepressed (Figure 4(e)). The toggle switch network remained stable in this state even after IPTG was not present after completion of the pulse. Similar to the varying response times we saw for aTc pulses before, the transition from one state to another occurs more slowly for longer connecting channel lengths, although we observed switching from a low to high state in all cases (Figure 4(f)). Considering that many genetic circuits incorporate small molecule control elements, we believe that our chip could serve as a useful platform for testing new network designs for which it is necessary to explore the parameter space of DNA template concentration together with small molecule regulators.

\section{Discussion}

As synthetic biologists construct increasingly complex biomolecular circuits, the size of the parameter space governing these circuits becomes larger and more complex as well, requiring high-throughput and fast methods that can speed up the design-build-test cycle. Utilizing cell-free systems enables circuits to be optimized more rapidly than with traditional in vivo circuit construction and analysis since time-consuming molecular cloning steps are not needed. However, not all genetic networks can be tested in batch conditions and consequently need to be carried out in a system that mimics the dilutions which occur during cellular divisions. We therefore developed an automated CFPU that performs steady-state in vitro tx-tl reactions in high throughput, facilitating the characterization of biological parts and dynamic circuits. Each of the 280 on-chip chemostats can be programmed with a unique set of DNA templates. By precisely controlling the amount of DNA template attached to the surface of each unit cell, we can effectively scan a range of DNA concentrations that correlate with variable steadystate protein expression levels. Different levels of protein expression can also be achieved by modifying the geometry of a unit cell. On-chip reagent formulation by microfluidic PWM makes it possible to perform complex and fully automated screens and generate complex reaction conditions in real time. Combining the capacity to create custom reagent compositions with the potential to survey the DNA sequence and concentration space, the CFPU automates in vitro synthetic biology experiments.

We note certain features that could be modified depending on the downstream application. For example, the unit cell geometry had only a subtle effect on gene circuit dynamics. Therefore, a future version of the CFPU could contain a single unit cell geometry, increasing throughput with respect to DNA concentration and sequence space. Considering prior work [17], we included connecting channels of different lengths on this device but found that this was not an optimal approach for controlling dilution rates due to the complexity arising from a reaction mixture containing molecules with vastly different diffusion coefficients. In future versions of the CFPU, dilution rates could instead be dialed in using different exchange rates and active mixing. The throughput of the CFPU device can be further expanded by at least $50 \%$ by increasing the number of unit cells in each row. The size of the device is limited by the size of the glass slide to which the chip is bonded, and with the current design, there is less room to expand vertically but still significant space to expand horizontally. Additionally, programming of the pneumatic valves during the experiment could be developed to be more user-friendly. For this work, custom LabView scripts were written for each type of experiment with minimal userdefined inputs (Figure S7); however, key valve control sequences have already been defined as subroutines within the program. More specifically, parameters for different phases of the experiment are user-defined, but the number and types of phases are hardwired into the program. Therefore, it would be possible to restructure the program to allow the user to combine specified control sequences in 


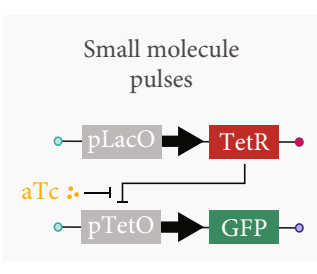

(a)
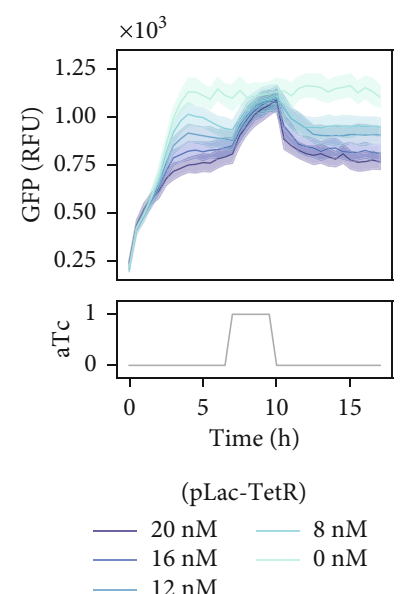

(b)

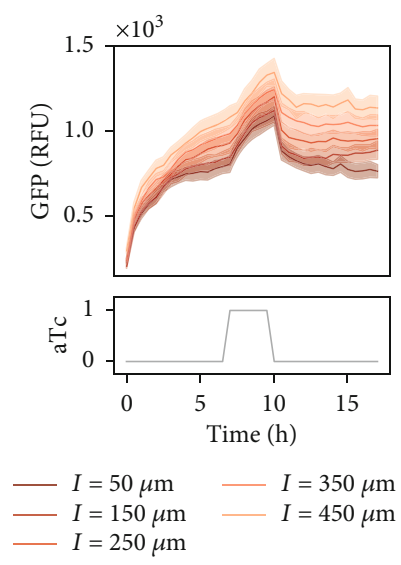

(c)

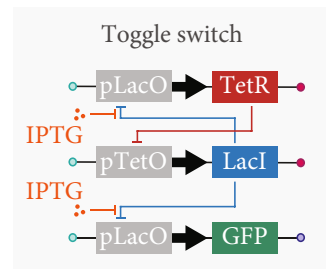

(d)
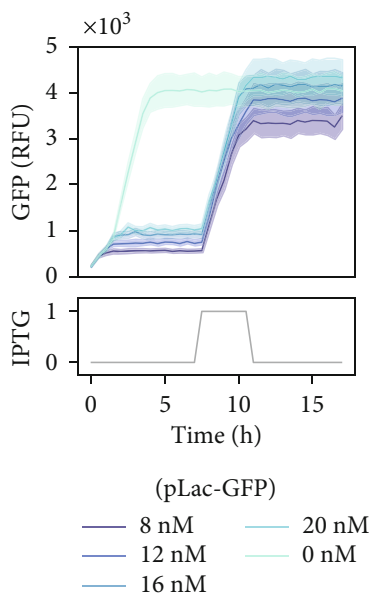

(e)

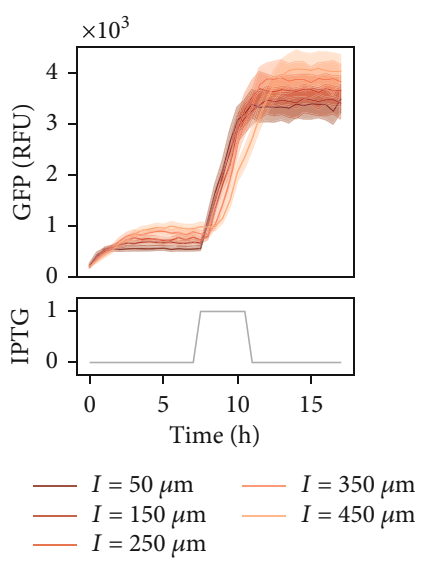

(f)

FIGURE 4: Small molecule perturbations and networks. (a) Illustration of the gene elements of the aTc controllable TetR repression assay. (b) GFP expression versus time for variable concentrations of pLacO-TetR DNA template spotted inside unit cells with a connecting channel length of $l=50 \mu \mathrm{m}$. (c) GFP expression profile over time shown for varying connecting channel lengths and a constant pLacO-TetR DNA template concentration equal to $20 \mathrm{nM}$. (d) Sketch of the toggle switch gene circuit. (e) GFP expression versus time displayed for a range of pLacO-GFP reporter concentrations spotted for unit cells with a connecting channel length of $l=50 \mu \mathrm{m}$. The concentration of the TetR and LacI templates are kept constant, giving a range of repressor to reporter template ratios from $1: 2$ to $3: 1$. (f) Time-lapse of GFP expression shown for different connecting channel lengths and a constant pLacO-GFP reporter concentration equal to $8 \mathrm{nM}$. All data presented in $(\mathrm{b}, \mathrm{c}, \mathrm{e}$, and $\mathrm{f})$ represents mean values $\pm \mathrm{SD}(n=8)$.

an interactive manner. Other features that allow the user to define reagent stock concentrations and desired dilutions could also be included to automatically calculate the duty cycles for all inputs.

Though the experimental setup behind the CFPU device is somewhat complex, it enables the study and characterization of dynamic gene circuits in a high-throughput and fully automated manner. Additional resources have been published that provide greater detail for fabricating two-layer PDMS devices and building hardware for controlling the on-chip pneumatic valves $[28,29]$. Apart from device fabrication and the experimental setup, another potential hurdle is access to a microarray robot. The use of a microarray robot is important for testing a large range of DNA template concentrations and variants. Over the last decade, microarray robots are increasingly less common, but still commercially available (Arrayit, Labnext, and Quanterix) and inkjetbased microarray printers offer a viable alternative.

In this work, we presented three ways that the CFPU device can be employed, which highlight key features that 
can be further exploited for many other applications (Figure S6). In the first experimental mode, we characterize the effect of DNA dilutions and unit cell geometry with continuous mixing of tx-tl reagents. In the second mode, we continuously supply tx-tl reagents, but once the reaction has reached a steady state, we switch the input mixture to contain a synthetic ZF transcription factor, which is screened for effective repression versus a number of promoter variants. Lastly, we demonstrate that a small molecule can be incorporated into the reaction mixture for a given duration, affecting the output of the surface immobilized gene circuit. Taken together, we show that the effect of changing the concentration and sequence of DNA parts can be investigated simultaneously with varying dilution rates and reagent inputs over time. That being said, the experimental modes we employed could be combined or expanded to explore the dynamics of gene circuits in a unique and programmable way. For instance, different concentrations of a molecule could be pulsed on-chip at sequential time points (Figure 1(i), bottom panel). Furthermore, the input could be programmed to respond to the gene circuit output, meaning that a new input formulation would only be generated if the reporter expression either exceeded or fell below a certain threshold, creating a fully automated, closed-loop system.

\section{Materials and Methods}

4.1. Microfluidic Chip Fabrication. The designs for the flow and control layer of the device were drawn with AutoCAD software. The device design files are available for download from our lab website (http://lbnc.epfl.ch) and http:// metafluidics.org. We then used standard photolithography to fabricate the molds for each layer. SU-8 negative photoresist was used to create the control channel features (GM 1070, Gersteltec Sarl) with a height of $30 \mu \mathrm{m}$, while AZ 9260 positive photoresist (Microchemicals $\mathrm{GmbH}$ ) was used to generate flow channel features with a height of $15 \mu \mathrm{m}$. Afterwards, each of the wafers was treated with TMCS (trimethylchlorosilane) and coated with PDMS (polydimethylsiloxane, Sylgard 184, Dow Corning). For the control layer, $\sim 50 \mathrm{~g}$ of PDMS with an elastomer to crosslinker ratio of $5: 1$ was prepared, whereas for the flow layer, a $20: 1$ ratio of elastomer to crosslinker was spin-coated at $1800 \mathrm{rpm}$ to yield a height of $\sim 50 \mu \mathrm{m}$. Both PDMS-coated wafers were then partially cured for 20 minutes at $80^{\circ} \mathrm{C}$, after which devices from the control layer were cut out and the inlets for each control line were punched. Each control layer is then aligned onto the flow layer by hand using a Nikon stereo microscope. The aligned devices were then placed at $80^{\circ} \mathrm{C}$ for 90 minutes, allowing the two layers to bond so that the entire device can be cut and removed from the flow wafer. Once the molds have been fabricated, it takes around three hours to make up to four two-layer PDMS devices at once.

4.2. Cell-Free Extract Preparation. E. coli cell-free extract was prepared according to a published method [30], and a $4 \mathrm{x}$ energy solution was prepared based on the protocol described by Sun et al. [31]. As Kwon et al. screened a num- ber of different parameters, we will briefly describe the protocol we have used. Cells were cultured with 2xYTP medium. After an initial overnight $5 \mathrm{~mL}$ culture, $1 \mathrm{~mL}$ of the overnight culture was added to a $500 \mathrm{~mL}$ Erlenmeyer flask with $200 \mathrm{~mL}$ of fresh medium. Four $200 \mathrm{~mL}$ cultures were incubated at $37^{\circ} \mathrm{C}$ until an OD of $\sim 2$ at $600 \mathrm{~nm}$ was reached. Each culture was then separated into falcon tubes and centrifuged at $4^{\circ} \mathrm{C}$ for 20 minutes at $4000 \mathrm{rpm}$. Cells were resuspended with a wash buffer $(10 \mathrm{mM}$ Tris, $14 \mathrm{mM}$ magnesium glutamate, $60 \mathrm{mM}$ potassium glutamate, and $2 \mathrm{mM}$ DTT) and centrifuged again at $4^{\circ} \mathrm{C}$ for 10 minutes at $4000 \mathrm{rpm}$. Following three wash steps, all excess liquid is removed, and the weight of each pellet is measured. The pellets are then flash-frozen in liquid nitrogen and stored at $-80^{\circ} \mathrm{C}$. The following day, the cell pellets are thawed on ice and resuspended with $1 \mathrm{~mL}$ wash buffer per gram of cell pellet. Sonication was used to lyse the cells by applying a total energy input of $\sim 400 \mathrm{~J}$ for a total cell suspension volume of $\sim 1-1.5 \mathrm{~mL}$. Cells were sonicated in an ice water bath with an amplitude of $50 \%$ with $10 \mathrm{~s}$ pulses interrupted by $10 \mathrm{~s}$ wait periods (Vibra-cell 75186 sonicator). Next, the lysate solutions are centrifuged at $4^{\circ} \mathrm{C}$ for 10 minutes at $12000 \times \mathrm{g}$. The supernatant is removed and placed at $37^{\circ} \mathrm{C}$ for 60 minutes to perform the run-off reaction. Lastly, the solution is centrifuged again at $4^{\circ} \mathrm{C}$ for 10 minutes at $12000 \times \mathrm{g}$, and the supernatant is then aliquoted into $50 \mu \mathrm{L}$ volumes, flash frozen, and stored at $-80^{\circ} \mathrm{C}$.

We prepared two different cell-free extracts originating from BL21 (DE3) and MC4100 cell strains. Lysates made from BL21 (DE3) cells were used for DNA dilution and ZF repression experiments, whereas MC4100 cell lysates were used for the small molecule inducible circuits as that strain is Lac- [32].

4.3. Preparation of Linear DNA Templates. Linear DNA templates were generated by assembly PCR in order to link a downstream gene with a given promoter variant. The methods for generating the $\mathrm{p} \lambda \mathrm{PR}-\mathrm{GFP}$ reporter templates used in the ZF repression assays have been previously described [15]. Gene-specific PCR fragments were obtained for TetR and LacI from the repressilator plasmid [32] and combined thereafter with promoter PCR fragments that contained either the Lac or Tet operons, respectively. Using global primers, we tagged each linear template at the $3^{\prime}$ end with biotin and at the $5^{\prime}$ end with either Cy 3 or Cy5.

4.4. Setting Up High-Throughput Steady-State Cell-Free $t x$ - $t$ l Experiments. The process of DNA spotting, chip alignment, and bonding, followed by chip priming, surface patterning, and DNA immobilization, has been described before [15]. A detailed protocol for the surface patterning was provided by Rockel et al. [33]. The total time required to complete these steps is approximately one day, including the overnight incubation for chip bonding. Once the DNA templates had been immobilized at the surface of the unit cells, the button valve was actuated, and the chip was washed with PBS for 15 minutes to ensure that any unbound DNA was removed and the DNA spot was visualized with fluorescence microscopy (Nikon Ti-SH-U). Before the experiment 
was started, the button valve was released and the sandwich valves were closed.

A lysate solution was prepared by combining lysate with wash buffer in a 1:1 ratio, and the energy solution was made up of $50 \% 4 \mathrm{x}$ energy solution, $2.5 \mu \mathrm{M}$ chi decoy DNA [34] or $3.5 \mu \mathrm{M}$ purified gamS [35], and water. The total volume of each solution should be between 30 and $40 \mu \mathrm{L}$ in order to run the reaction for 15-20 hours. Each solution was drawn into a separate piece of FEP tubing (OD 1/16", ID 1/32", Upchurch) and connected to the microfluidic device inlet with a small piece of PEEK tubing (OD 1/32", ID $0.18 \mathrm{~mm}$, Vici). The pressure applied to the lysate and energy solution tubing was $\sim 20 \mathrm{kPa}$ during the experiment. Every 15 minutes, a fresh mixture of cell-free extract was flowed through the exchange channel using a cycle period of $600 \mathrm{~ms}$ and a duty cycle of $50 \%$ for 100 total cycles (or $\sim 60$ s) to ensure that the entire channel volume has been replaced (Figure S1F, G). To mix three components for the ZF repression experiment, a cycle period of $1 \mathrm{~s}$ was used for a total of 60 cycles to result in the same overall flow time. Before the cell-free mixture was flowed, valve (1) was opened and valve (2) was closed, followed by a $10 \mathrm{~s}$ wait period to assure that valve (1) opened completely in all rows (Figure 1(a)). Once the exchange channel was replaced with fresh reagents, valve (1) was closed and valve (2) was released permitting the diffusion of reaction components into and out of the unit cells. This process of exchange followed by incubation was carried out continuously for up to 20 hours while the chip was maintained in a temperaturecontrolled box set to $33^{\circ} \mathrm{C}$. GFP expression was monitored in all unit cells over time by acquiring a fluorescence image every 30 minutes.

Switching from one cell-free mixture to another could be programmed. For instance, if a pulse of aTc or IPTG should be delivered to the unit cells at a given time, then three solutions were prepared: (1) a lysate solution, (2) an energy solution, and (3) an energy solution plus either $5 \mu \mathrm{M}$ of aTc or $250 \mu \mathrm{M}$ of IPTG. During the small molecule pulse, the cellfree mixture will be generated from solutions (1) and (3), whereas at all other times, it will be produced from solutions (1) and (2). For the ZF experiment, three solutions were also used: (1) a lysate solution, (2) a lysate solution plus $5 \mu \mathrm{M}$ ZF$\mathrm{mScarlet}$, and (3) an energy solution. Initially, solutions (1) and (3) are used to reach steady-state GFP expression levels; then, all three solutions are used to generate different concentrations of ZF-mScarlet within the cell-free mixture. Furthermore, the multiplexing valves are actuated accordingly to replace each exchange channel with a cell-free mixture containing a predefined ZF concentration. To ensure that the dead volume inside the serpentine mixing channel does not affect the next mixture generated, a PBS wash step is included every time the duty cycle percentage changes.

4.5. Experimental Automation. A custom LabView program was written to control the pneumatic valves, the camera, and the microscope (Figure S7). The on-chip pneumatic valves were controlled by opening and closing electric solenoid valves that are connected to regulated air pressure. The LabView program allows the user to either toggle a valve on or off manually or run a specific valve sequence. Although the surface patterning and immobilization of DNA templates could be automated, it was performed manually in order to ensure that no air bubbles entered the chip when flowing new solutions. Once the DNA was attached to the surface and all necessary inputs were plugged into the chip, a custom sequence was initiated enabling the valves to actuate automatically throughout the course of the entire experiment. Different scripts were written for each type of experiment performed. For instance, when performing basic characterization experiments, the user defines the cycle period, the duty cycle for each of the two solutions (lysate and energy), and the total number of cycles, corresponding to the amount of time the mixture should be flowed to replace the exchange channel. Additionally, the user defines the incubation time between each flow step and the total number of exchange/incubate cycles that should be performed based on the desired length of the experiment. Therefore, steady-state gene expression can be performed autonomously on-chip as shown in Figures $1(\mathrm{f})$ and $1(\mathrm{~g})$. If the experiment involved switching from one mixture to another at a designated time, such as introducing a $\mathrm{ZF}$ or supplying a small molecule pulse, then a different script was written to allow for different experimental phases. The first phase followed the same script as for the basic characterization experiments. The second phase follows the same concept; however, the user must now define the duty cycle for each of the three solutions to be mixed. Depending on the duty cycle chosen, a given concentration of ZF or small molecule will be formulated on-chip. When small molecule pulses were generated during an experiment, the script would then include a third phase that is the same as the first. The user defines the total amount of time for each phase. If the experiment involved multiplexing, as with the ZF combinatoric experiments, then it was hardwired into the specific script rather than being user-defined.

Given that imaging and valve actuation occurred at different frequencies, control of the valves was independent from that of the camera and the microscope in the LabView program. In order to image the unit cells over time, the user is required to indicate the total experiment time and imaging frequency, as well as the desired imaging parameters, including exposure time and fluorescence filter. Additionally, the positions of the top left, top right, and bottom right unit cells need to be saved. The program then calculates the horizontal and vertical distances used to move the stage from one unit cell to the next when imaging.

\section{Data Availability}

All data used to support this study are included in the article and the supplementary information files (CFPU).

\section{Conflicts of Interest}

The authors declare no conflict of interest. 


\section{Authors' Contributions}

SZ and SJM developed the project. ZS performed experiments. ZS and SJM designed experiments, analyzed data, and wrote the manuscript.

\section{Acknowledgments}

We thank Ivan Istomin and Amir Shahein for providing us with the purified ZF-mScarlet protein. This work was supported by the European Research Council under the European Union's Horizon 2020 research and innovation program Grant 723106 (SJM) and SNF Project grant 182019 (SJM).

\section{Supplementary Materials}

Figure S1: characterization of PWM for on-chip mixing of lysate and energy solutions. Figure S2: disrupting steadystate expression. Figure S3: chip-to-chip reproducibility. Figure S4: GFP expression for a range of DNA dilutions. Figure S5: mixing 3 components on-chip with PWM. Figure S6: summary of CFPU experiments. Figure S7: overview of the LabView program. (Supplementary Materials)

\section{References}

[1] D. Sprinzak and M. B. Elowitz, "Reconstruction of genetic circuits," Nature, vol. 438, no. 7067, pp. 443-448, 2005.

[2] C. J. Bashor and J. J. Collins, "Understanding biological regulation through synthetic biology," Annual Review of Biophysics, vol. 47, no. 1, pp. 399-423, 2018.

[3] N. Laohakunakorn, L. Grasemann, B. Lavickova et al., "Bottom-up construction of complex biomolecular systems with cell-free synthetic biology," Frontiers in Bioengineering and Biotechnology, vol. 8, 2020.

[4] A. S. Khalil and J. J. Collins, "Synthetic biology: applications come of age," Nature Reviews Genetics, vol. 11, no. 5, pp. 367-379, 2010.

[5] D. E. Cameron, C. J. Bashor, and J. J. Collins, "A brief history of synthetic biology," Nature Reviews Microbiology, vol. 12, no. 5, pp. 381-390, 2014.

[6] P. A. Silver, J. C. Way, F. H. Arnold, and J. T. Meyerowitz, "Engineering explored," Nature, vol. 509, no. 7499, pp. 166$167,2014$.

[7] A. D. Silverman, A. S. Karim, and M. C. Jewett, "Cell-free gene expression: an expanded repertoire of applications," Nature Reviews Genetics, vol. 21, no. 3, pp. 151-170, 2020.

[8] M. K. Takahashi, J. Chappell, C. A. Hayes et al., "Rapidly characterizing the fast dynamics of RNA genetic circuitry with cell-free transcription-translation (TX-TL) systems," ACS Synthetic Biology, vol. 4, pp. 503-515, 2015.

[9] D. Siegal-Gaskins, Z. A. Tuza, J. Kim, V. Noireaux, and R. M. Murray, "Gene circuit performance characterization and resource usage in a cell-free \breadboard," ACS Synthetic Biology, vol. 3, no. 6, pp. 416-425, 2014.

[10] H. Niederholtmeyer, Z. Z. Sun, Y. Hori et al., "Rapid cell-free forward engineering of novel genetic ring oscillators," eLife, vol. 4, article e09771, 2015.

[11] S. J. Moore, J. T. MacDonald, S. Wienecke et al., "Rapid acquisition and model-based analysis of cell-free transcription- translation reactions from nonmodel bacteria," Proceedings of the National Academy of Sciences of the United States of America, vol. 115, pp. 4340-4349, 2018.

[12] A. Swaminathan, V. Hsiao, and R. M. Murray, Quantitative Modeling of Integrase Dynamics Using a Novel Python Toolbox for Parameter Inference in Synthetic Biology, bioRxiv, 2017.

[13] Y. Hori, C. Kantak, R. M. Murray, and A. R. Abate, "Cell-free extract based optimization of biomolecular circuits with droplet microfluidics," Lab on a Chip, vol. 17, no. 18, pp. 30373042, 2017.

[14] J. Fan, F. Villarreal, B. Weyers et al., "Multi-dimensional studies of synthetic genetic promoters enabled by microfluidic impact printing," Lab on a Chip, vol. 17, no. 13, pp. 2198 2207, 2017.

[15] Z. Swank, N. Laohakunakorn, and S. J. Maerkl, "Cell-free generegulatory network engineering with synthetic transcription factors," Proceedings of the National Academy of Sciences of the United States of America, vol. 116, no. 13, pp. 5892-5901, 2019.

[16] H. Niederholtmeyer, V. Stepanova, and S. J. Maerkl, "Implementation of cell-free biological networks at steady state," Proceedings of the National Academy of Sciences of the United States of America, vol. 110, no. 40, pp. 15985-15990, 2013.

[17] E. Karzbrun, A. M. Tayar, V. Noireaux, and R. H. Bar-Ziv, "Programmable on-chip DNA compartments as artificial cells," Science, vol. 345, no. 6198, pp. 829-832, 2014.

[18] A. Adamo, R. L. Beingessner, M. Behnam et al., "On-demand continuous-flow production of pharmaceuticals in a compact, reconfigurable system," Science, vol. 352, no. 6281, pp. 61-67, 2016.

[19] L. E. Crowell, A. E. Lu, K. R. Love et al., "On-demand manufacturing of clinical-quality biopharmaceuticals," Nature Biotechnology, vol. 36, no. 10, pp. 988-995, 2018.

[20] K. S. Boles, K. Kannan, J. Gill et al., "Digital-to-biological converter for on-demand production of biologics," Nature Biotechnology, vol. 35, no. 7, pp. 672-675, 2017.

[21] N. Hartrampf, A. Saebi, M. Poskus et al., "Synthesis of proteins by automated flow chemistry," Science, vol. 368, no. 6494, pp. 980-987, 2020.

[22] K. Woodruff and S. J. Maerkl, "Microuidic module for realtime generation of complex multimolecule temporal concentration Profiles," Analytical Chemistry, vol. 90, pp. 696-701, 2017.

[23] J.-C. Chang, Z. Swank, O. Keiser, S. J. Maerkl, and E. Amstad, "Microfluidic device for real-time formulation of reagents and their subsequent encapsulation into double emulsions," Scientific Reports, vol. 8, no. 1, p. 8143, 2018.

[24] T. Thorsen, S. J. Maerkl, and S. R. Quake, "Microfluidic largescale integration," Science, vol. 298, no. 5593, pp. 580-584, 2002.

[25] B. Lavickova, N. Laohakunakorn, and S. J. Maerkl, A selfregenerating synthetic cell model, bioRxiv, 2020.

[26] M. C. Blackburn, E. Petrova, B. E. Correia, and S. J. Maerkl, "Integrating gene synthesis and microfluidic protein analysis for rapid protein engineering," Nucleic Acids Research, vol. 44, no. 7, article e68, 2016.

[27] T. S. Gardner, C. R. Cantor, and J. J. Collins, "Construction of a genetic toggle switch in Escherichia coli," Nature, vol. 403, no. 6767, pp. 339-342, 2000.

[28] A. J. van der Linden, M. Yelleswarapu, P. A. Pieters et al., "A multilayer microuidic platform for the conduction of 
prolonged cell-free gene expression," Journal of Visualized Experiments, no. 152, article e59655, 2019.

[29] N. Laohakunakorn, B. Lavickova, Z. Swank, J. Laurent, and S. J. Maerkl, "Steady-state cell-free gene expression with microuidic chemostats," in Synthetic Gene Circuits : Methods and Protocols, F. Menolascina, Ed., pp. 189-203, Springer US, New York, NY, USA, 2021.

[30] Y.-C. Kwon and M. C. Jewett, "High-throughput preparation methods of crude extract for robust cell-free protein synthesis," Scientific Reports, vol. 5, no. 1, article 8663, 2015.

[31] Z. Z. Sun, C. A. Hayes, J. Shin, F. Caschera, R. M. Murray, and V. Noireau, "Protocols for implementing an Escherichia coli based TX-TL cell-free expression system for synthetic biology," Journal of Visualized Experiments, no. 79, article e50762, 2013.

[32] M. B. Elowitz and S. Leibler, "A synthetic oscillatory network of transcriptional regulators," Nature, vol. 403, no. 6767, pp. 335-338, 2000.

[33] S. Rockel, M. Geertz, and S. J. Maerkl, "MITOMI: a microuidic platform for in vitro characterization of transcription factorDNA interaction," in Gene Regulatory Networks: Methods and Protocols, Methods in Molecular Biology, B. Deplancke and N. Gheldof, Eds., pp. 97-114, Humana Press, Totowa, NJ, USA, 2012.

[34] R. Marshall, C. S. Maxwell, and S. P. Collins, "Short DNA containing $\chi$ sites enhances DNA stability and gene expression in E. coli cell-free transcription-translation systems," Biotechnology and Bioengineering, vol. 114, no. 9, pp. 2137-2141, 2017.

[35] Z. Z. Sun, E. Yeung, C. A. Hayes, V. Noireaux, and R. M. Murray, "Linear DNA for rapid prototyping of synthetic biological circuits in an Escherichia coli based TX-TL cellfree system," ACS Synthetic Biology, vol. 3, no. 6, pp. 387397,2014 